\title{
Leveraging Elasticsearch to Improve Data Discoverability in Science Gateways
}

\author{
Jake Rosenberg \\ jrosenberg@tacc.utexas.edu \\ Texas Advanced Computing Center \\ Austin, TX
}

\author{
Josue Balandrano Coronel \\ jcoronel@tacc.utexas.edu \\ Texas Advanced Computing Center \\ Austin, TX
}

\author{
Joseph Meiring \\ jmeiring@tacc.utexas.edu \\ Texas Advanced Computing Center \\ Austin, TX
}

\author{
Sarah Gray \\ sgray@tacc.utexas.edu \\ Texas Advanced Computing Center \\ Austin, TX
}

\author{
Tracy Brown \\ tbrown@tacc.utexas.edu \\ Texas Advanced Computing Center \\ Austin, TX
}

\begin{abstract}
Data discoverability is a challenge in science gateway architectures. As the volume of data managed and shared through a science gateway grows, it is imperative to expose a search functionality which enables users to quickly navigate to files within their own data sets as well as to identify relevant files in shared or public data sets. Desirable qualities in a file search feature include scalability to arbitrary data sizes, rapid and responsive indexing triggered by user activity, and easy maintainability by development teams without specialist knowledge of search algorithms. We describe a search architecture built around Elasticsearch that meets each of these criteria, and which has been successfully implemented at the Texas Advanced Computing Center to enhance data discoverability in several science gateway projects.
\end{abstract}

\section{CCS CONCEPTS}

- Information systems $\rightarrow$ RESTful web services.

\section{KEYWORDS}

Science Gateways, Elasticsearch

\section{ACM Reference Format:}

Jake Rosenberg, Josue Balandrano Coronel, Joseph Meiring, Sarah Gray, and Tracy Brown. 2019. Leveraging Elasticsearch to Improve Data Discoverability in Science Gateways. In Practice and Experience in Advanced Research Computing (PEARC '19), July 28-August 1, 2019, Chicago, IL, USA. ACM, New York, NY, USA, 5 pages. https://doi.org/10.1145/3332186.3332230

\section{INTRODUCTION}

The Texas Advanced Computing Center (TACC) maintains an expanding portfolio of science gateway projects aimed at connecting researchers in diverse domains with TACC's high-performance computing (HPC) resources. Recent additions include gateways

Permission to make digital or hard copies of all or part of this work for personal or classroom use is granted without fee provided that copies are not made or distributed for profit or commercial advantage and that copies bear this notice and the full citation on the first page. Copyrights for components of this work owned by others than ACM must be honored. Abstracting with credit is permitted. To copy otherwise, or republish, to post on servers or to redistribute to lists, requires prior specific permission and/or a fee. Request permissions from permissions@acm.org.

PEARC '19, fuly 28-August 1, 2019, Chicago, IL, USA

(c) 2019 Association for Computing Machinery.

ACM ISBN 978-1-4503-7227-5/19/07 . \$ \$15.00

https://doi.org/10.1145/3332186.3332230 for the NeuroNex Technology Hub, Planet Texas 2050, and the UT Research Cyberinfrastructure. These projects all share the goal of facilitating the curation, processing, and sharing of data by users. By providing a web interface for data curation and submission of jobs to HPC systems, we are able to provide advanced computing capabilities to users without specialist knowledge of HPC architectures. We also provide new features, such as interactive applications, which are unavailable to users with only command-line access.

Each science gateway project at TACC provides a graphical user interface which allows researchers to manage files on Corral, a highperformance replicated storage resource maintained by TACC. This interface is powered by the Agave API. Agave is a TACC-maintained science-as-as-service platform which provides RESTful access to HPC resources [3]. In the science gateway context, Agave provides metadata and permission information for all user-managed files on Corral. Users can navigate their folders and upload, download, move, copy, and preview files according to their permissions. Data sharing is accomplished through a concept called Projects, in which a user can define a virtual directory to share and manage access by collaborators. As the volume of data shared using these gateways grows, a key architectural challenge and request from our users is to make this data quickly and efficiently discoverable from the user interface. As the volume of data grows and with some projects generating tens of millions of files, the discoverability of data has often been a hindrance to the science goals of a project, especially in a collaborative environment. We describe our efforts to enhance data discoverability at TACC using Elasticsearch, a search engine and document store which provides the capability to analyze and search file metadata.

\section{ELASTICSEARCH: BACKGROUND AND SCIENCE GATEWAY IMPLEMENTATION}

Elasticsearch is a document store and search engine based on Apache Lucene. Hallmark features include facile horizontal scalability and the ability to query documents in near-realtime once they have been indexed.[2] Elasticsearch is deployed as a cluster containing one or more nodes which serve the indexed data. Data indexed in the cluster is sharded across the nodes in a redundant fashion, and the number of shards and replicas can be tuned for performance. Documents that share a metadata schema comprise an index within the cluster. For example, science gateway projects 


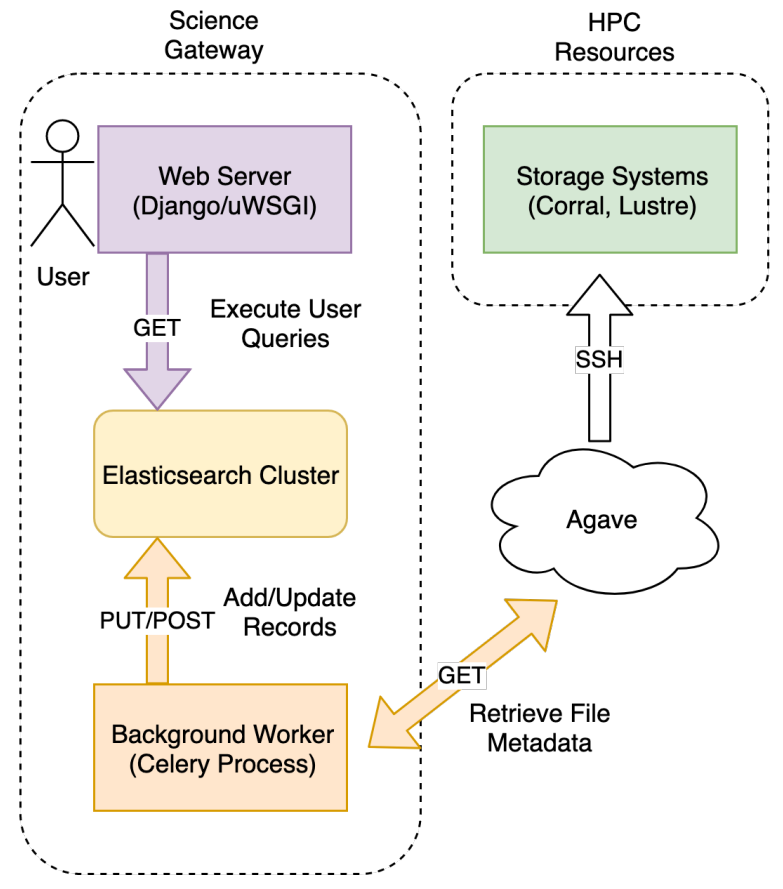

Figure 1: Search architecture for a science gateway interacting with HPC resources through Agave.

at TACC use separate Elasticsearch indices for data files, collaborative projects, and supplementary web content. The index definition contains information about what fields are expected to be contained in the indexed metadata- e.g. the filename, path, and user permissions for files; or the PI and authorized collaborators for a project. The index definition also contains analyzers which are associated with metadata fields. An analyzer is a set of rules governing how the metadata indexed in a particular field should be tokenized for search purposes. Custom analyzers are defined as a combination of a character filter, tokenizer, and token filter, which are applied sequentially. The character filter transforms text prior to tokenization, the tokenizer uses a set of rules to split the text into tokens, and the token filter applies additional transformations to each generated token.

Any time a new document is indexed into the Elasticsearch cluster, the analysis rules for each field are applied and a set of tokens is generated. These tokens are placed into an inverted index, which relates each token to the document which produced it. The use of an inverted index allows for extremely fast searches, as a query string can be quickly matched against the ordered set of tokens without having to perform any additional sorting or processing of the indexed data. If no analyzer is specified for a metadata field, Elasticsearch uses a standard analyzer, which splits text into tokens at whitespace and non-alphanumeric characters according to the Unicode text segmentation guidelines.

Searches against documents indexed in Elasticsearch are performed by making HTTP requests against a REST API exposed by the cluster. Queries are performed using a domain-specific language (DSL) which allows for complex combinations of filters and Boolean

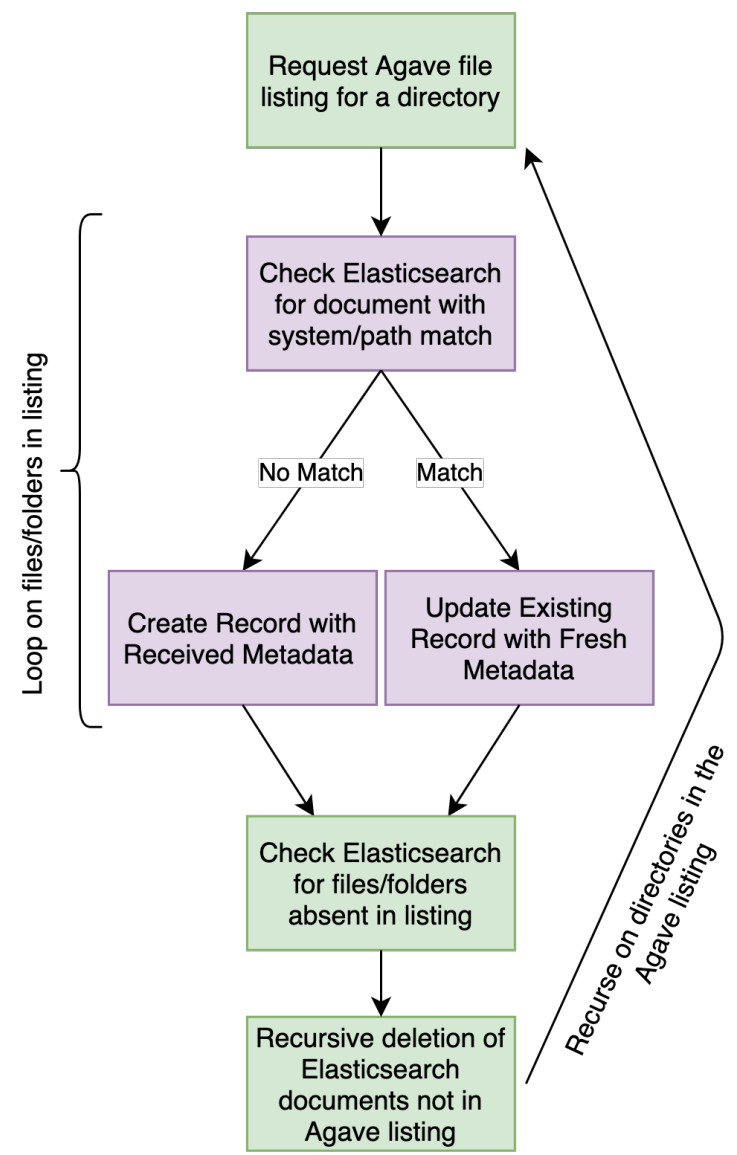

Figure 2: Flowchart detailing the file indexing process in a science gateway.

logic. The Elasticsearch API also supports creation, modification, and deletion of documents through POST, PUT, and DELETE requests respectively. Each index can be associated with one or more aliases. An API request targeting an index's alias is automatically redirected to that index. Aliases can be added or removed through the REST API, and several of these operations can be combined in a single atomic transaction; we will demonstrate how this feature is helpful for performing bulk updates to existing indices.

In the science gateway architecture being developed at TACC, each gateway is deployed alongside a single-node Elasticsearch cluster running on the same virtual machine as the web server. This cluster contains only the documents relevant to its associated science gateway. The port exposing the API service is secured behind a firewall in order to prevent manipulation of the indices by unauthorized users. Search is exposed to users via a search bar in the data browser interface. When a user executes a search, their query string input is matched against the tokens in the inverted index, with the results filtered based on permissions and the storage system which the user wishes to query. Any matches are displayed using the same interface used to show data files.

The Elasticsearch architecture implemented at TACC Is diagrammed in Figure 1. When a user executes a search, the query is 
processed within the web server's request/response cycle due to the short response time enabled by the inverted index structure (50-100 ms). Tasks which add or update documents in the Elasticsearch index are performed using a background worker process in order to avoid blocking requests while file metadata is retrieved. In order to populate the Elasticsearch index with discoverable files, we "walk" the directory structure of a user's storage system using calls to the Agave API.[1] Agave retrieves metadata for files and folders in a single directory at a time by establishing an SSH connection to the HPC resource containing user or project data. This metadata is used to update the Elasticsearch index. Subsequently, the worker process spawns a new task which lists files and folders in any directories discovered during the initial listing. This recursive process continues until the full directory structure of a user's storage system has been indexed.

When the indexer process receives file metadata from Agave, it executes a series of steps which ensure parity between the Elasticsearch index and the file system which it represents. A flow chart of the indexer's operation is given in Figure 2. For each file and folder metadata object retrieved from Agave, there is an initial check to determine whether a document representing this object already exists in the Elasticsearch index. A file can be uniquely identified using a combination of its "system" and "path" attributes. If the Elasticsearch index contains a document with matching values for those attributes, then that document is retrieved and refreshed with updated metadata. Otherwise, a new document is created and populated. This check ensures that a single file in a storage resource is never associated with multiple Elasticsearch documents. If multiple documents with the same system/path combination are discovered at this time, all but one of these documents is deleted. The remaining document is then refreshed with the latest metadata from Agave, so that there is no risk of deleting a fresh version of a document while retaining a stale one. Once each metadata object in the Agave listing has been indexed, the final step is to check for files or folders which are present in Elasticsearch but absent on the storage resource. This is the stage at which we account for files which have been deleted or moved to a different directory. If documents matching this criterion are discovered, then they are deleted. This prevents searches from returning "ghost" hits for files which can no longer be accessed at their originally indexed paths. If the document being deleted represents a folder, the Elasticsearch index is walked recursively in order to remove any documents corresponding to data within that folder. With this done, parity is achieved between the metadata retrieved from Agave and the metadata indexed in the Elasticsearch cluster. If the Agave listing contained no folders, then the indexer task exits; otherwise, the indexer asynchronously spawns a new task to index each folder.

Because indexing a large storage system with hundreds or thousands of subdirectories necessarily involves an equal number of requests to the Agave API under this implementation, the indexing process has been tuned to minimize API traffic where possible. Typically, full storage system indexing occurs only when onboarding a new user or setting up a new project. Afterwards, when the indexer is dispatched due to an action taken by a user within the science gateway interface, only files affected by that action are listed and indexed. In these cases, the indexer can be called with a flag which prevents it from recursively spawning additional tasks.

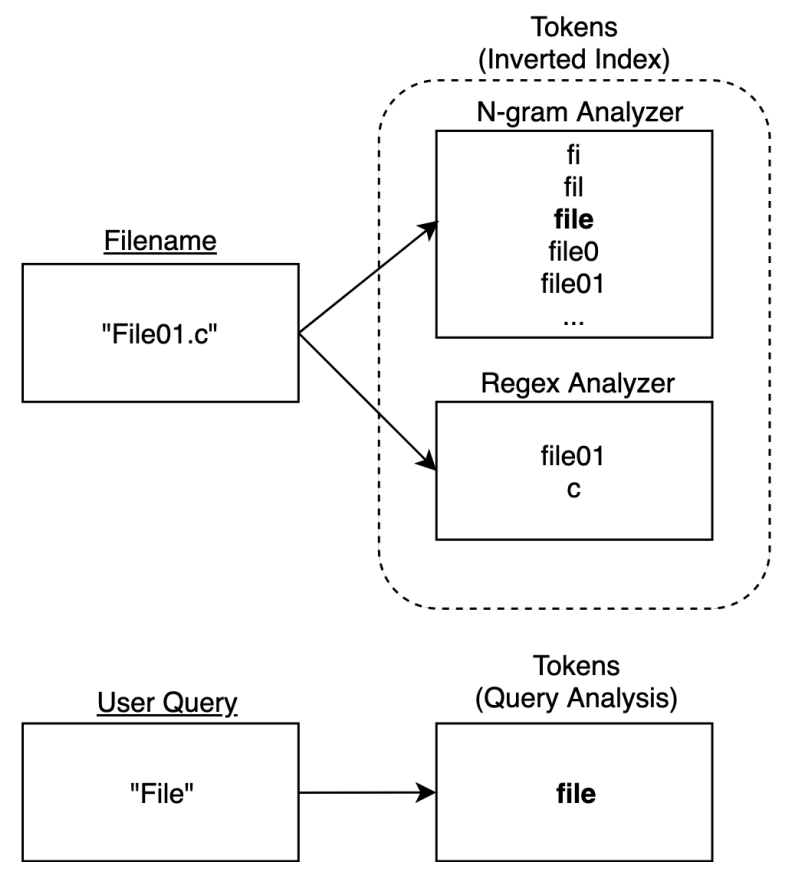

Figure 3: Example of search on filenames. The bolded tokens represent a match between the analyzed search query and the inverted index.

For example, if a text file is moved from one directory to another, file metadata is retrieved only for files and folders in the origin and destination directories, without recursing further into directories unaffected by the change. If a folder is moved, however, in addition to non-recursive indexing of the source and destination directories, there is a need to recursively index the folder in its new location in order to ensure that the indexed documents contain updated path and permission metadata.

\section{FILENAME ANALYSIS AND SEARCH}

When a file's metadata is indexed into Elasticsearch, each field is analyzed in order to populate the inverted index with tokens that can be matched on search operations. Most fields are analyzed as simple keywords, meaning that the text they contain is placed directly into the inverted index without applying filters or tokenizers. This is appropriate for fields such as unique IDs or permissions strings, which require an "all-or-nothing" approach to matching queries. Filenames, however, present an exception in that it is important to permit matches even if a user does not input the full text. In well-curated research data, filenames should contain considerable information on the content and experimental context of the data.[4] It is therefore crucial to the data discoverability problem that a query on some relevent keyword should return hits on any file containing that keyword in its name. In our search implementation, partial matching is facilitated by the use of a custom analyzer employing an n-gram tokenizer. The tokenizer is configured to generate tokens containing each continuous sequence of between 2 and 20 characters within a filename. A filter is applied to lower-case each token so that searches are case-insensitive. 


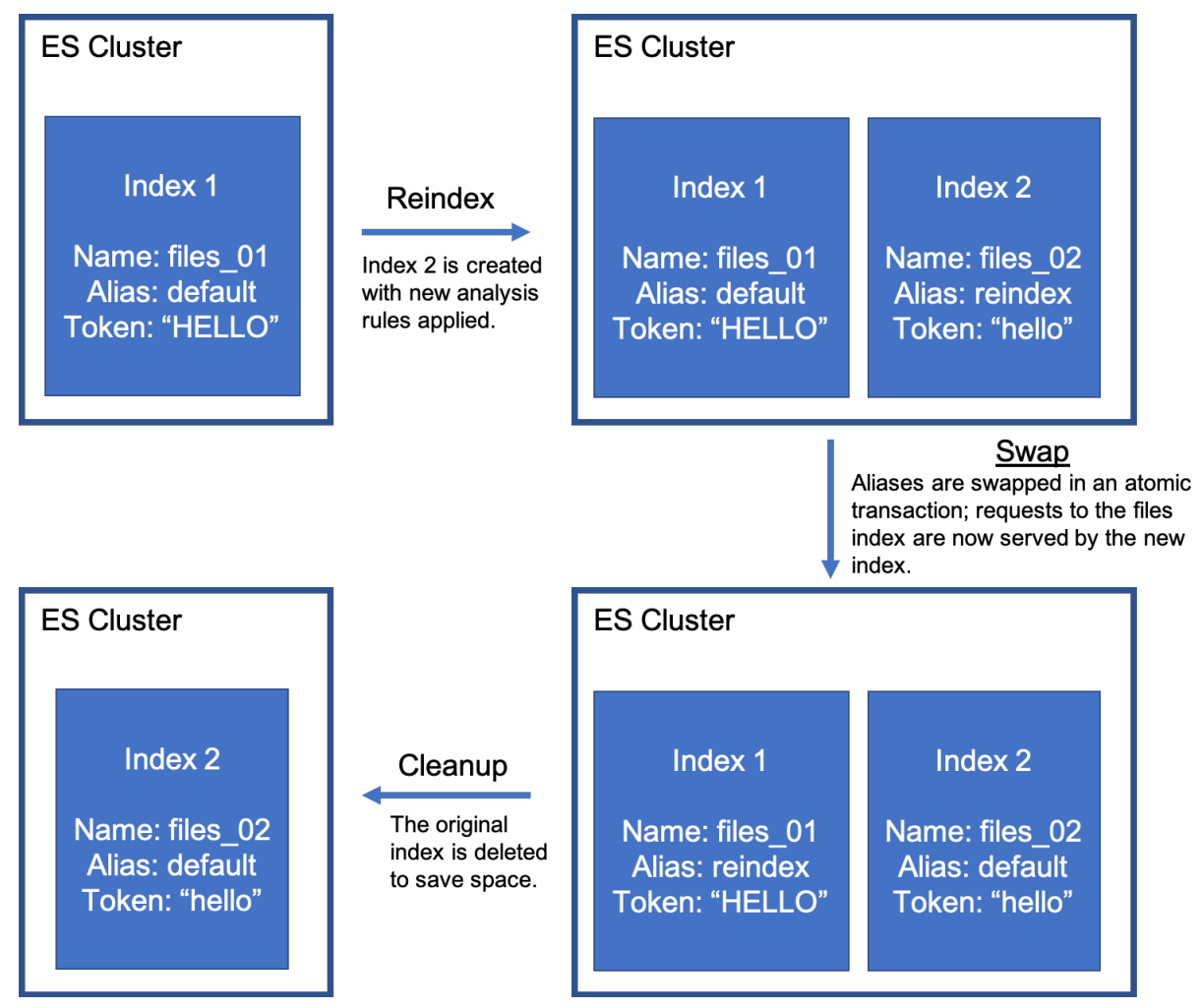

Figure 4: Schematic representation of zero-downtime reindexing within a science gateway.

When a user performs a search, their query string is analyzed using a separate keyword-based analyzer. This analyzer lower-cases the query string, but performs no additional operations. The query string token is then searched against the n-gram tokens in the inverted index in order to find a match. Thus, any query string which matches a sequence of 2 or more characters in a filename produces a hit which is displayed to the user in the science gateway interface. This process is shown visually in Figure 3.

A special consideration has to be made for query strings containing over 20 characters. These strings, when analyzed as keywords, generate tokens over 20 characters long, and therefore cannot generate exact matches against the n-grams in Elasticsearch's inverted index. Increasing the n-gram size in these cases is impossible, since analysis of indexed files occurs only at index time. Our solution for these long query strings is to apply a token filter which truncates the keywords to a maximum of 20 characters, so that a match against the first 20 characters is sufficient to return a hit.

Another edge case which must be accounted for is that of a onecharacter query with relevance to the user. For instance, a search on a file extension such as "c" would be expected to return results for files ending in the .c extension, or a search for "a" would be expected to match the file "a.out". We can generalize this example to queries on files for which either the name or extension consists of a single character. To address this case, a second analyzer is deployed which uses a regular expression to split indexed filenames on the full stop character, creating tokens containing the text on either side. This solution is preferable to the alternative of lowering the minimum n-gram size to one, both because it saves space in the Elasticsearch cluster and because it avoids making the search overly permissive. Allowing matches on single character n-grams would generate massive numbers of irrelevant hits on single-character queries, while this regex solution provides more targeted hits.

\section{REINDEXING AND MAINTENANCE CONSIDERATIONS}

When considering the implementation of search functionality across several science gateway projects, it was necessary to design an implementation which would be easily maintainable across several deployments in different research domains. Specifically, we required a mechanism to add new fields or analyzers needed in a specific deployment. Updating an index often involves re-analyzing all documents in that index in order to generate a new inverted index with the correct tokens. This process can take minutes to hours depending on the number of documents which need to be reindexed; therefore, we were faced with a need to implement a solution that did not affect search functionality during the reindexing process. Additionally, these search deployments need to be maintained by different teams of developers with varying levels of experience with the Elasticsearch internals.

In order to address the issue of reindexing quickly and without downtime, a command-line utility was developed to instantiate and update indices based on the configuration of a particular science gateway. The reindexing strategy is shown schematically in Figure 
4. A new index is first instantiated which contains the same fields and analyzer definitions as the index which is to be updated. This new index has a name which is timestamped according to its date of creation, and an alias which denotes that it is intended to be used for reindexing. Once the new index has been created, all documents in the extant index are copied into the "reindexing" index. During this process, they are automatically re-analyzed with the most current iteration of the index mapping. Once the new index has been fully populated, the aliases of the new and old indexes are swapped in a single atomic transaction through the Elasticsearch REST API. Because all search operations in TACC-maintained science gateways are performed based on aliases rather than true names, swapping the aliases in this fashion instantly causes all search queries to target the new alias. There is zero downtime in the sense that there is no point at which a search initiated by a science gateway user does not return results from a fully populated index. Using this utility, the search schema for a new metadata type can be quickly propagated across several gateway projects; alternately, a domain-specific search update can be implemented in a single science gateway by developers without specialist knowledge of Elasticsearch.

\section{CONCLUSIONS AND FUTURE WORK}

Elasticsearch, in combination with the Agave API and TACC's HPC resources, provides a search solution which enhances data discoverability in science gateways. We have developed a search infrastructure which is easily deployable and highly maintainable, even by developers without specific search expertise. Future work will focus on taking advantage of Elasticsearch's first-class support for distributed document storage. We plan to shard our existing indices between multiple virtual machines provisioned for this purpose. This will ensure reliability in the case of hardware failure, and enhance the speed of searches as the volume of stored metadata expands into the terabyte scale.

In order to increase indexing throughput and reduce API traffic to Agave, we plan to refactor our indexer to take advantage of the Abaco platform developed at TACC. Abaco provides functionsas-a-service by registering Docker images as actors which can be executed on HPC hardware.[5] Rather than requiring a discrete API request for each folder being indexed, a proposed Abaco indexer would require only a single connection to be established to the storage system being indexed. Once the connection is established, an Abaco reactor could walk the directory structure while harvesting metadata, intermittently sending webhook requests to the science gateway backend to trigger indexing in Elasticsearch.

\section{ACKNOWLEDGMENTS}

We thank the Cloud and Interactive Computing group at TACC for their development and support of the Agave platform.

\section{REFERENCES}

[1] Josue Balandrano Coronel and Stephen Mock. 2017. DesignSafe: Using Elasticsearch to Share and Search Data on a Science Web Portal. In Proceedings of the Practice and Experience in Advanced Research Computing 2017 on Sustainability, Success and Impact - PEARC17. ACM Press, New Orleans, LA, USA, 1-3. https://doi.org/10.1145/3093338.3093386

[2] Manda Sai Divya and Shiv Kumar Goyal. 2013. ElasticSearch 'An advanced and quick search technique to handle voluminous data'. Compusoft 2, 6 (2013), 171-175.
[3] Rion Dooley, Dan Stanzione, Steve Terry, and Edwin Skidmore. 2012. Software-as-aservice: the iPlant foundation API. In 5th IEEE Workshop on Many-Task Computing on Grids and Supercomputers (MTAGS).

[4] Lisa Johnston (Ed.). 2017. Curating research data. Volume one: Practical strategies for your digital repository. Association of College and Research Libraries, a division of the American Library Association, Chicago, Illinois.

[5] Joe Stubbs, Walter Moreira, Matthew Vaughn, Julia Looney, Rion Dooley, Julian Pistorius, Ehb54, and Ke'Darius Whitley. 2018. TACC/abaco: August 2018. https: //doi.org/10.5281/zenodo.1924546 